\title{
Genetically modified human placenta-derived mesenchymal stem cells with FGF-2 and PDGF-BB enhance neovascularization in a model of hindlimb ischemia
}

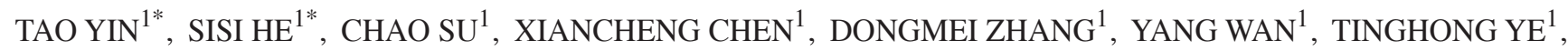 \\ GUOBO SHEN $^{1}$, YONGSHENG WANG ${ }^{1,2}$, HUASHAN SHI $^{1}$, LI YANG $^{1}$ and YUQUAN WEI ${ }^{1,2}$ \\ ${ }^{1}$ State Key Laboratory of Biotherapy and Cancer Center, West China Hospital, Sichuan University, \\ and Collaborative Innovation Center for Biotherapy, Chengdu, Sichuan 610041; ${ }^{2}$ Department of Thoracic Oncology, \\ Cancer Center, West China Hospital, Sichuan University, Chengdu, Sichuan 610041, P.R. China
}

Received December 1, 2013; Accepted June 26, 2015

DOI: $10.3892 / \mathrm{mmr} .2015 .4089$

\begin{abstract}
Ischemic diseases represent a challenging worldwide health burden. The current study investigated the therapeutic potential of genetically modified human placenta-derived mesenchymal stem cells (hPDMSCs) with basic fibroblast growth factor (FGF2) and platelet-derived growth factor-BB (PDGF-BB) genes in hindlimb ischemia. Mesenchymal stem cells obtained from human term placenta were transfected ex vivo with adenoviral bicistronic vectors carrying the FGF2 and PDGF-BB genes (Ad-F-P). Unilateral hindlimb ischemia was surgically induced by excision of the right femoral artery in New Zealand White rabbits. Ad-F-P genetically modified hPDMSCs, Ad-null (control vector)-modified hPDMSCs, unmodified hPDMSCs or media were intramuscularly implanted into the ischemic limbs 7 days subsequent to the induction of ischemia. Four weeks after cell therapy, angiographic analysis revealed significantly increased collateral vessel formation in the Ad-F-P-hPDMSC group compared with the control group. Histological examination revealed markedly increased capillary and arteriole density in the Ad-F-P-hPDMSC group. The xenografted hPDMSCs survived
\end{abstract}

Correspondence to: Dr Li Yang or Dr Yuquan Wei, State Key Laboratory of Biotherapy and Cancer Center, West China Hospital, Sichuan University, and Collaborative Innovation Center for Biotherapy, 17 People's South Road, Chengdu, Sichuan 610041, P.R. China

E-mail: yl.tracy73@gmail.com

E-mail: yqwei@vip.sina.com

*Contributed equally

Abbreviations: FGF2, basic fibroblast growth factor; PDGF-BB, platelet-derived growth factor-BB; hPDMSCs, human placenta-derived mesenchymal stem cells; Ad-F-P, adenoviral bicistronic vectors carrying FGF2 and PDGF-BB genes

Key words: ischemia, angiogenesis, arteriogenesis, gene therapy in the ischemic tissue for at least 4 weeks subsequent to cell therapy. The current study demonstrated that the combination of hPDMSC therapy with FGF2 and PDGF-BB gene therapy effectively induced collateral vessel formation and angiogenesis, suggesting a novel strategy for therapeutic angiogenesis.

\section{Introduction}

Ischemic diseases remain a challenging problem of clinical relevance, despite advances in relevant medical intervention. A number of patients with cardiovascular or peripheral ischemia are not suitable candidates for conventional revascularization procedures. For those with no alternative, therapeutic angiogenesis has been indicated to be a promising treatment strategy. Several successful experimental and clinical trials of pro-angiogenic therapy have been conducted, with genes such as hypoxia-inducible factor (1), nerve growth factor (2) and placental growth factor (3). Since the natural process of angiogenesis is complex and multi-factorial, single factor-based therapy often fails to promote the formation of mature and stable vasculature, and may even have detrimental effects. Sustained expression of vascular endothelial growth factor (VEGF) has been demonstrated to lead to extensive edema and destroy the normal organ architecture (4). Therefore, the combination of different pro-angiogenic factors, including VEGF and fibroblast growth factor (FGF) (5), granulocyte-colony stimulating factor and hepatocyte growth factor (6), and VEGF and monocyte chemoattractant protein 1 (7) has also been investigated.

Basic FGF (FGF2) is a powerful mitogen in a variety of cell types, including endothelial and smooth muscle cells (8), and has been used as a stimulator of angiogenesis and arteriogenesis (9). Platelet-derived growth factor (PDGF) has the ability to recruit smooth muscle cells and participate in arteriogenesis (10). Blood vessel formation not only requires endothelial cells, but also pericytes and smooth muscle cells (11). The synergistic pro-angiogenic effect of FGF2 and PDGF-BB in the revascularization process have previously been reported $(12,13)$, and when simultaneously administered into ischemic tissues, the two factors can promote mature and stable vessel formation. 
Cell therapy is another promising therapeutic approach to ischemic tissue regeneration and repair $(14,15)$. The use of mesenchymal stem cells (MSCs) in angiogenic stem cell transplantation has been widely exploited, due to characteristics such as easy isolation and expansion. MSCs can undergo multipotent differentiation in vivo and in vitro, can home to and incorporate into sites of neovascularization, secrete angiogenic factors and promote neovacularization through paracrine mechanisms $(16,17)$. Due to its unique feature of little or low immunogenicity, MSCs can be administered without the requirement of human leukocyte antigen (HLA) matching (18). MSCs, isolated from bone marrow and adipose tissue and expanded in vitro, have already been used for therapeutic angiogenesis $(16,19)$. However, the paucity of these cells is hampering their application. Along with easy accessibility and abundant MSCs, the placenta has proven to be an attractive cell source for cell therapy (20-22). Additionally, MSCs isolated from the placenta have similar biological characteristics to those from the bone marrow (23). Previous studies have indicated that hPDMSCs can be used for the treatment of ischemic diseases (24-27).

In the present study, hPDMSCs were isolated and expanded in vitro, and transfected with adenoviral bicistronic vectors carrying the FGF2 and PDGF-BB genes. It was hypothesized that this strategy of a combination of gene therapy and stem cell therapy may more effectively enhance neovascularization compared with previously tested angiogenic factors.

\section{Materials and methods}

Harvest, culture and isolation of hPDMSCs. A term gestation placenta from a healthy donor mother was obtained with informed consent from the West China Second Hospital (Chengdu, China). Placental tissues were harvested and washed several times with low-glucose Dulbecco's modified Eagle's medium (DMEM; Gibco Life Technologies, Carlsbad, CA, USA) under sterile conditions. It was then minced into a coarse slurry with scissors in a Petri dish, followed by enzymatic digestion with $1 \mathrm{mg} / \mathrm{ml}$ collagenase (Sigma-Aldrich, St. Louis, MO, USA) for $\sim 2 \mathrm{~h}$ at $37^{\circ} \mathrm{C}$. The homogenate was centrifuged at $188 \mathrm{x} \mathrm{g}$ for $3 \mathrm{~min}$. The predispositions were suspended and cultured in the T75 flasks with low-glucose DMEM supplemented with $20 \%$ fetal bovine serum (Gibco Life Technologies). Cell cultures were maintained in a humidified $5 \% \mathrm{CO}_{2}$ atmosphere at $37^{\circ} \mathrm{C}$. Cells were identified by their typical fibroblast-like morphology under an AxioVert 200 inverted phase microscope (Zeiss, Thornwood, NY, USA). Under daily observation, the initial medium was changed $\sim 6$ days after plating, and nonadherent cells were removed. Thereafter, media were changed every 3 days. Cells were passaged at $80-90 \%$ confluence with $0.25 \%$ trypsin (Gibco Life Technologies).

Immunophenotyping of hPDMSCs. To detect the immunophenotype of hPDMSCs, flow cytometric analyses were performed. Aliquots of cells were incubated with the following antibodies: Monoclonal anti-human CD29 (cat. no. MA1-82635; Thermo Fisher Scientific, Inc., Waltham, MA, USA); monoclonal anti-human CD90 (cat. no. MA1-24985; Thermo Fisher Scientific, Inc.); monoclonal anti-human CD105 (cat. no. MS-1290-P; Thermo Fisher Scientific, Inc.); monoclonal anti-human CD31 (cat. no. ab9498-500; Abcam, Cambridge, MA, USA), followed by fluorescein isothiocyanate- and phycoerythrin-conjugated secondary anti-mouse IgG antibodies (cat. nos. 11-4011 and 12-4010; eBioscience, Inc., San Diego, CA, USA). Isotype-identical antibodies were used as controls. Labeled cells were acquired by flow cytometry using a BD FACSCalibur cell analyzer and Cellquest software 6.0 (BD Biosciences, San Jose, CA, USA).

Adenoviral bicistronic vector construction and transfection of hPDMSCs. hPDMSCs at passages 5-8 were used in the current experiments. Adenoviral bicistronic vectors were constructed containing human FGF2 and PDGF-BB genes, in which those two genes were separated by a cis-acting region designated internal ribosome entry site element. Therefore, the vectors could secrete both FGF2 and PDGF-BB. Ad-null vectors were constructed without the FGF-2 and PDGF-BB genes. The cells were cultured at a density of $2 \times 10^{6}$ cells $/ 75 \mathrm{~cm}^{2}$ tissue culture flask and incubated with Ad-F-P or Ad-null at a multiplicity of infection of 1,500 with the addition of Lipofectamine ${ }^{\circledR} 2000$ (Invitrogen Life Technologies, Carlsbad, CA, USA) when cells were at $70-80 \%$ confluence. Following transduction for $4 \mathrm{~h}$, cells were incubated for another $48 \mathrm{~h}$ prior to the in vitro experiment and cell transplantation.

Hindlimb ischemia model and cell delivery. All animal interventions were performed in accordance with guidelines of the Sichuan University Institutional Animal Care and Use Committee (Chengdu, China). Male New Zealand White rabbits (4-5 months old and 2.5-3.0 kg in weight; Animal Experimental Center of the West China Hospital, Sichuan, China) were used to produce a hindlimb ischemia model. Briefly, the rabbits were anesthetized with $3 \%$ pentobarbital sodium $(30 \mathrm{mg} / \mathrm{kg}$; Animal Experimental Center of West China Hospital), the femoral artery of the right hindlimb was exposed and freed from the inguinal ligament to the point where it bifurcates into the popliteal and saphenous arteries. All branches were ligated prior to excision of the whole femoral artery. A total of 20,000 U/kg penicillin (Shiyao Pharmaceutical Group Co., Ltd., Shijiazhuang, China) were administered intramuscularly for 3 days subsequent to surgery. Seven days after unilateral femoral artery excision, one of the following was injected into the right ischemic adductor muscles ( $\mathrm{n}=5$ in each group): $5 \times 10^{6}$ Ad-F-P-hPDMSCs, 5x10 6 Ad-null-hPDMSCs, 5x106 hPDMSCs or low-glucose DMEM medium. After the initiation of cell therapy, observations of edema, inflammation, and limb and toe necrosis in the ischemic hindlimbs were performed daily.

Angiographic assessment. Four weeks subsequent to treatment, the animals were subjected to angiography. Briefly, under anesthetization, a catheter was inserted into the left femoral artery and advanced into the aorta. Serial filming of the right hindlimb was performed with a Philip-FD-201000 mA angiography machine (Philips, Amsterdam, Netherlands). Quantitative angiographic analysis was performed with previously described methods (28). Briefly, a composite of grids was placed over the ischemic area. The number of grid intersections that were crossed by opacified vessels was counted. The angiographic score in each film was calculated as the ratio 
A

B
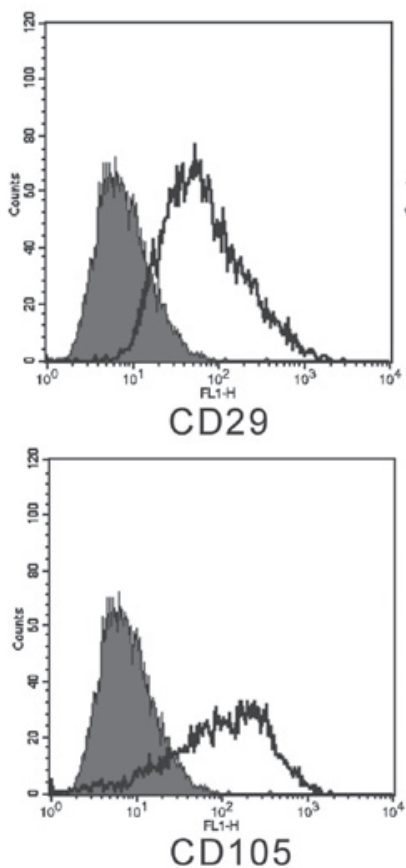

Figure 1. Morphology and immunophenotype of hPDMSCs. (A) hPDMSCs adhered to plastic and exhibited spindle-like morphology. (B) Typical MSC markers on hPDMSCs were evaluated by flow cytometry. hPDMSCs were positive for CD29, CD90 and CD105, and negative for CD31. hPDMSCs, human placenta-derived MSCs; MSC, mesenchymal stem cell.

of grid intersections crossed by opacified vessels divided by the total number of grid intersections in this area.

Immunohistochemical studies. Following angiography, the animals were sacrificed by air embolism, and the right adductor muscle tissues were harvested immediately and fixed in $4 \%$ paraformaldehyde or preserved at $-80^{\circ} \mathrm{C}$. The dissected adductor muscles were embedded in paraffin and sectioned at $5 \mu \mathrm{m}$. Hematoxylin and eosin (HE) staining was performed. To evaluate the density of capillaries and arterioles following cell therapy, sections were stained with mouse monoclonal anti-rabbitCD31 (1:100; cat.no. ab9498-500; Abcam) and mouse monoclonal anti-rabbit $\alpha$ smooth muscle actin ( $\alpha$ SMA) (1:100; cat. no. ab18147-250; Abcam). Identification of the survival of xenografted hPDMSCs was performed with mouse monoclonal primary antibody anti-human surface of intact mitochondria protein (1:800; cat. no. MAB1273; EMD Millipore, Billerica, MA, USA). Biotinylated polyclonal goat anti-mouse $\operatorname{IgG}$ was used as the secondary antibody (cat. no. PV-6002; ZSGB-BIO
A
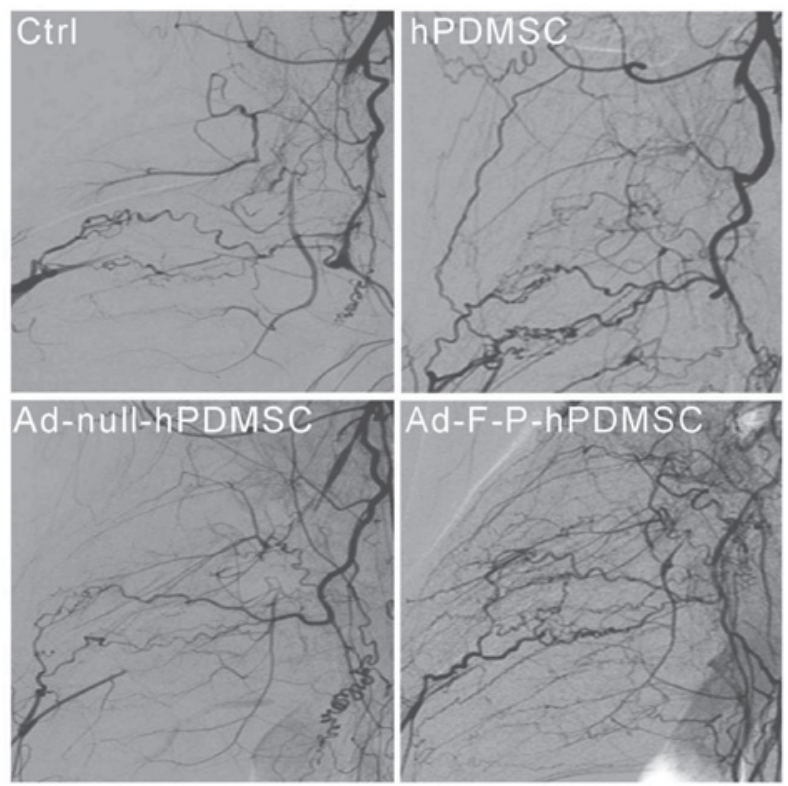

B

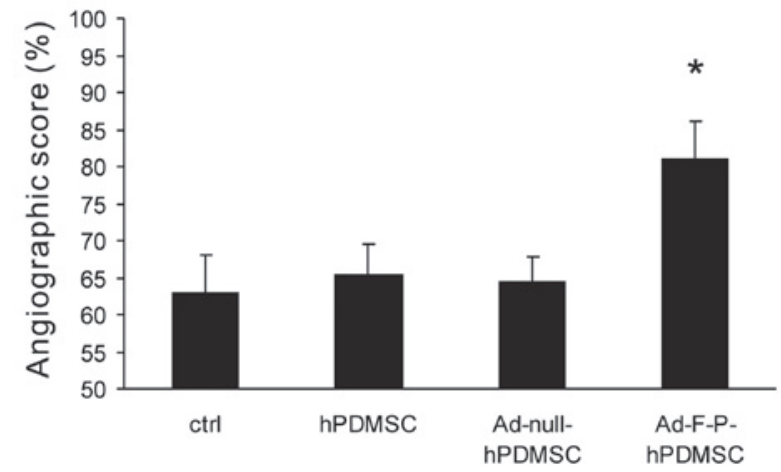

Figure 2. Angiographic assessment of collateral vessel formation. (A) Angiography was performed 28 days after cell therapy. More collateral vessels were visualized in the Ad-F-P-hPDMSC group. (B) Quantification of angiographic score. Genetically-modified hPDMSCs robustly enhanced collateral vessel formation. ${ }^{*} \mathrm{P}<0.05$ vs. control group. hPDMSC, human placenta-derived mesenchymal stem cell; AD-F-P, adenoviral bicistronic vector containing FGF2 and PDGF-BB; Ad-null, control vector; ctrl, control without PDMSCs.

ORIGENE, Beijing, China), followed by streptavidin-biotin horseradish peroxidase complex, and colorimetric detection was performed with diaminobenzidine (Fuzhou Maixin Biotechnology Development Co., Ltd., Fuzhou, China) supplemented with $0.03 \%$ hydrogen peroxide. Slides were counter-stained with hematoxylin solution, dehydrated and mounted. The capillary and arteriole densities were evaluated under a LEICA DM 2500 microscope (Leica Microsystems GmbH, Wetzlar, Germany).

Statistical analysis. All data are expressed as the mean \pm standard deviation. Statistical analysis was conducted with SPSS 13.0 software (SPSS, Inc, Chicago, IL, USA). Comparisons between groups were conducted by analysis of variance. $\mathrm{P}<0.05$ was considered to indicate a statistically significant difference.

\section{Results}

hPDMSCs have the typical characteristics of MSCs. Isolated cells were cultured on plastic in low-glucose DMEM selected 


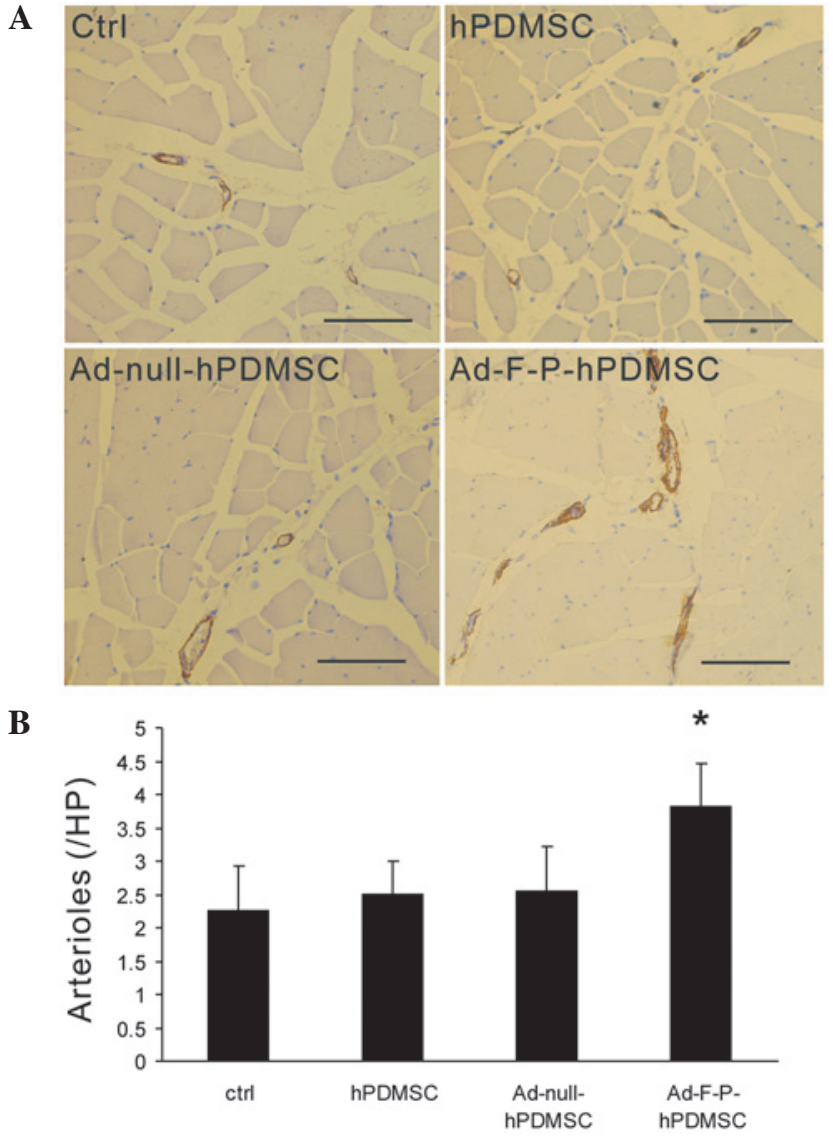

Figure 3. Assessment of mature arterioles. (A) Ischemic muscle sections were stained with antibody against $\alpha$ SMA to evaluate arteriole maturation. More aSMA + mural cell-covered arterioles were observed in Ad-F-P-hPDMSCs group. Scale bar, $50 \mu \mathrm{m}$. (B) Quantification of mature arterioles in ischemic muscles. Genetically-modified hPDMSCs significantly increased mature artery formation. ${ }^{*} \mathrm{P}<0.05$ vs. control. $\alpha$ SMA, $\alpha$ smooth muscle actin; hPDMSC, human placenta-derived mesenchymal stem cell; AD-F-P, adenoviral bicistronic vector containing FGF2 and PDGF-BB; Ad-null, control vector; ctrl, control without PDMSCs.

for MSCs outgrowth. Roughly 6 days subsequent to seeding, adherent cells in small colonies with a spindle-like phenotype were visualized. The cells presented a large expansive potential following subculture. The spindle-like morphology, as displayed in Fig. 1A, was maintained throughout the culture period. Immunophenotype examination was performed to further identify the hPDMSCs. hPDMSCs expressed CD29, CD90 and CD105, and were negative for CD31 (Fig. 1B).

Ad-F-P-hPDMSCs promote collateral vessel formation. To test whether genetically modified hPDMSCs were able to enhance collateral vessel formation in a model of ischemia, angiography was performed at 28 days subsequent to cell therapy. Greater collateral vessel formation was observed in the hindlimbs of Ad-F-P-hPDMSC-treated rabbits compared with those of other groups (Fig. 2A). Quantitative analysis of collateral vessels demonstrated that the angiographic score in the Ad-F-P-hPDMSC group was significantly higher than that of the control group (Fig. 2B). hPDMSCs alone did not significantly induce collateral vessel formation, although the average angiographic score was higher in the hPDMSC group compared with that of the control group. These results suggest
A
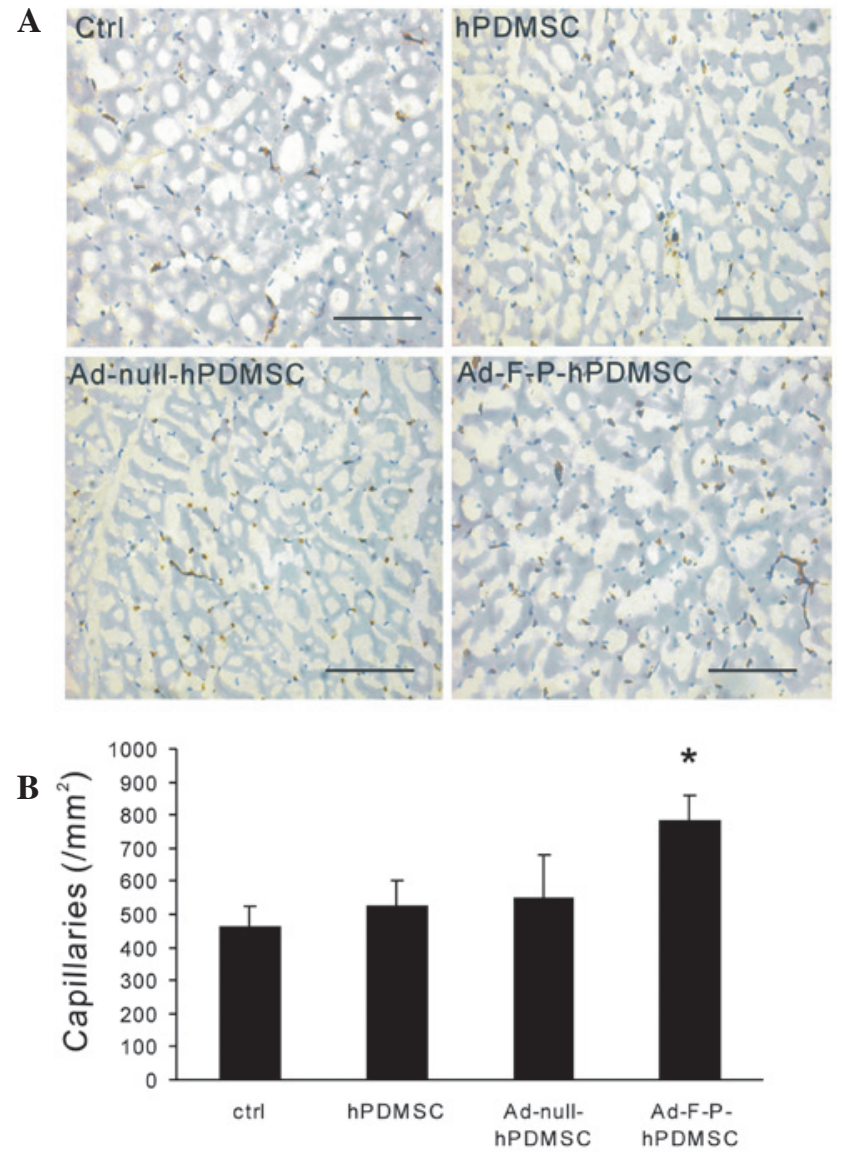

Figure 4. Assessment of capillary density. (A) Ischemic muscle sections were stained with an antibody against CD31 to evaluate capillary density. A greater density of capillaries was observed in the Ad-F-P-hPDMSC group. Scale bar, $50 \mu \mathrm{m}$. (B) Quantification of capillary density in ischemic muscles. ${ }^{*} \mathrm{P}<0.05$ vs. control. hPDMSC, human placenta-derived mesenchymal stem cell; AD-F-P, adenoviral bicistronic vector containing FGF2 and PDGF-BB; Ad-null, control vector; ctrl, control without PDMSCs.

that the delivery of genetically-modified hPDMSCs with FGF2 and PDGF-BB has a greater ability to induce robust arteriogenesis compared with hPDMSCs alone.

Ad-F-P-hPDMSCs increase mature artery formation. FGF2 and PDGF have been demonstrated to establish stable and mature vessels associated with recruitment of mural cells (12). To further determine whether Ad-F-P-hPDMSCs are able to promote mature arteriole formation, muscle sections were stained with antibodies against $\alpha$ SMA. The arteriole density was higher in the Ad-F-P-hPDMSC group compared with that of other groups (Fig. 3A). An increase in aSMA-covered vessels was observed in the Ad-F-P-hPDMSC group. However, there was no significant difference between the Ad-null-hPDMSC, hPDMSC and control groups in the arteriole density (Fig. 3B). These results suggest that Ad-F-P-hPDMSCs promote arteriole maturation in ischemic areas.

Ad-F-P-hPDMSCs increase capillary formation. To further evaluate the impact of genetically modified hPDMSCs on the formation of capillaries in the ischemic area, histological examination of ischemic adductor muscle was performed. Capillary density in the Ad-F-P-hPDMSC group was higher than in other groups (Fig. 4). However, no significant difference 

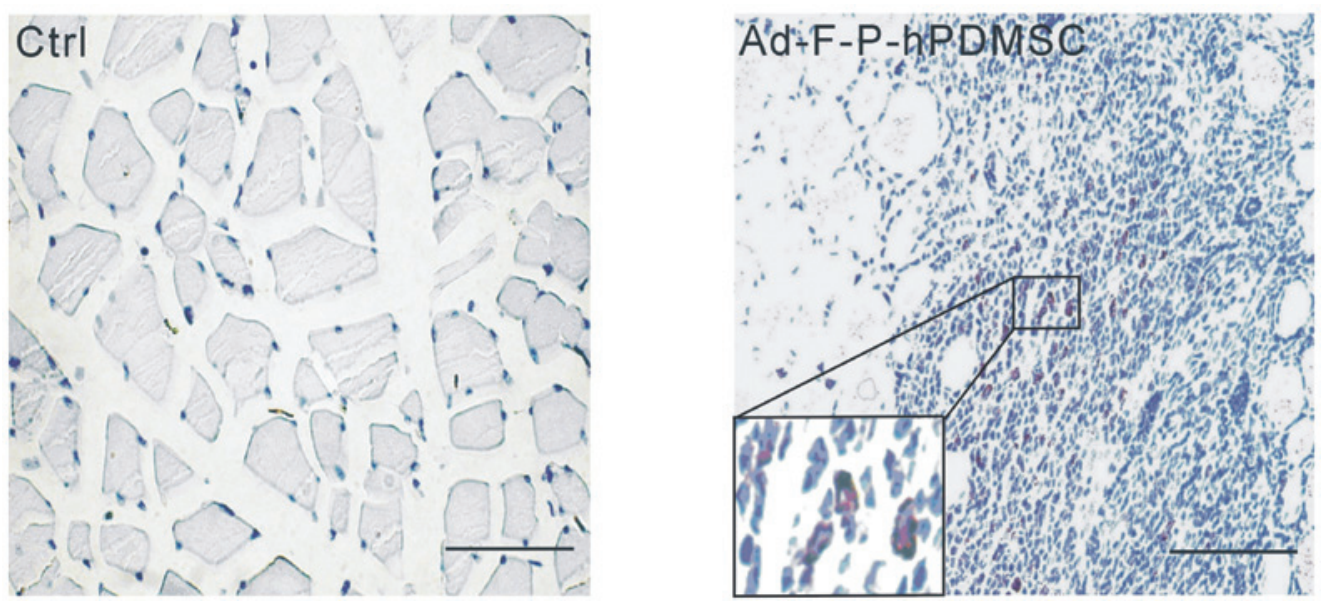

Figure 5. hPDMSC survival in the ischemic tissue. Immunohistochemical staining with an antibody against human-specific surface of intact mitochondria protein indicated that xenografted hPDMSCs survived in the ischemic tissue for at least four weeks. Scale bar, $50 \mu \mathrm{m}$; magnification $\mathrm{x}$. hPDMSC, human placenta-derived mesenchymal stem cell. AD-F-P, adenoviral bicistronic vector containing FGF2 and PDGF-BB; ctrl, control without PDMSCs.

was detected between the Ad-null-hPDMSC, hPDMSC and control groups.

Xenografted hPDMSCs survive in ischemic areas. H\&E staining was performed to detect the pathological changes in the ischemic muscles. No muscular atrophy or hemangioma was detected in any of the groups. In the Ad-F-P-hPDMSC group, numerous large cells aggregated in the intermuscular area. To evaluate whether the cells were transplanted $e x$ vivo, sections were immunostained with a specific antibody against human mitochondria. The results indicated that several cells were positive for a human-specific marker (Fig. 5). These results suggest that the xenotransplanted hPDMSCs existed for at least 4 weeks in the ischemic muscle.

\section{Discussion}

In the present study, MSCs from human term placenta (hPDMSCs) that had a spindle-like, plastic-adherent phenotype, were isolated. Flow cytometry suggested that hPDMSCs expressed CD29, CD90 and CD105, but not CD31. The hPDMSCs were genetically modified with adenoviral bicistronic vectors that carried the FGF2 and PDGF-BB genes simultaneously. Local delivery of the genetically modified hPDMSCs into the adductor muscles of the ischemic hindlimb significantly enhanced collateral formation and increased the capillary and arteriole density. Therefore, a combination of FGF2 and PDGF-BB gene therapy with hPDMSC therapy is able to enhance neovascularization, which requires angiogenesis and arteriogenesis.

hPDMSCs were genetically modified to overexpress FGF2 and PDGF-BB in the ischemic muscle tissue. A greater level of capillary and arteriole formation was observed in the Ad-F-P-hPDMSC group. FGF2 is a powerful stimulator of angiogenesis and arteriogenesis. As the most pluripotent form of PDGF (28), PDGF-BB is an effective stimulator of qsmooth muscle cell proliferation and migration, and participates in the process of arteriogenesis. The synergistic effects of FGF2 and PDGF-BB in the revascularization process have previously been reported in different animal models of angiogenesis $(12,13)$. Reciprocal interplay between receptor signaling systems contributes to their pro-angiogenic synergism. FGF2 upregulates PDGFR expression in endothelial cells; PDGF-BB induces the release of FGF2 and activation of FGFR-1, and upregulates FGFR-1 expression in vascular smooth muscle cells $(12,29,30)$.

In addition, compared with other factors, the combination of FGF2 and PDGF-BB achieves the strongest pro-angiogenic effect (11). The protein infusion and gene delivery of FGF2 and PDGF-BB have already been used to enhance therapeutic neovascularization, and can promote angiogenesis and arteriogenesis. However, compared with previously used combinatorial factors, a bicistronic vector carrying FGF2 and PDGF-BB genes together was constructed in the present study, which simplified therapeutic procedures.

Stem cell therapy provides a promising alternative and adjuvant therapy to current pro-angiogenic therapy (31). Currently, the mechanisms of therapeutic angiogenesis with MSCs have been demonstrated to involve two aspects as follows: MSCs secrete a wide spectrum of angiogenic and arteriogenic cytokines, and enhance neovascularization by a paracrine mechanism (16). Additionally, MSCs differentiate into endothelial and smooth muscle cells in the ischemic tissues, and enhance neovascularization directly (32). hPDMSCs have been reported to secrete a large amount of bioactive VEGF (33). However, in the present study, no significant improvement was observed in collateral vessel formation, which may be attributable to the low power of hPDMSCs. In the present study, genetically modified PDMSCs had greater efficacy than pure hPDMSCs with regards to therapeutic angiogenesis. To achieve a similar magnitude of therapeutic neovascularization, cell therapy alone requires 30 times more cells than ex vivo-transduced cells (34). It is possible that the administration of a higher density of hPDMSCs is required to achieve a similar effect as was created with Ad-F-P-hPDMSCs. The combination of exogenous genes of angiogenic factors with the MSCs may be able to achieve superior revascularization (35).

Since systemic intravenous delivery of MSCs can be limited by the entrapment of donor cells in the lung $(36,37)$, a method involving local implantation of hPDMSCs was used, 
and a single therapy was indicated to be sufficient to augment neovasculariztion. A previous study indicated that premature cessation of VEGF administration results in regression of acquired vessels (4). Unlike VEGF, one dose of FGF2 and PDGF-BB can maintain functional and stable vessels (12).

MSCs have unique immunological characteristics and persist in a xenogeneic or allogeneic environment (38). In the present study, the xenotransplanted hPDMSCs survived in the ischemic muscle tissues for at least 4 weeks, without the use of immunosuppressive drugs. A previous study suggested that immunological tolerance of xenografted amniotic membrane-derived MSCs may be due to HLA-G expression, and the activation of regulatory $\mathrm{T}$ cells (39). A previous study confirmed that hPDMSCs have low immunogeneity (25), therefore it is possible that this low immunogeneity and the ability to tolerate the low oxygen concentration contribute to the survival of hPDMSCs in ischemic tissues. These properties suggest that hPDMSCs can act as a durable source of FGF2 and PDGF-BB secretion following implantation into ischemic tissue, which may produce relatively long-lasting expression of potent factors. Ex vivo transduction may preclude the exposure of adenovirus to the host immune system, then MSC-based cell therapy may ameliorate the adverse effect caused by adenovirus (40). In the present experiment, Ad-F-P-hPDMSCs was able to secrete long-lasting pro-angiogenic factors.

There are several limitations to the current study. The fate of the MSCs was not determined in vivo, but MSCs have previously been demonstrated to integrate into neovasculature directly and differentiate into endothelial cells or smooth muscle cells (32). Although it was indicated in the present study that the xenografted cells can survive in the ischemic areas, the molecular mechanisms were not elucidated. Further investigation is required to clarify these remaining questions.

In conclusion, the present study demonstrated the following: (i) Human placenta represents a promising source of MSCs, and hPDMSCs are an alternative vector for gene therapy; (ii) local delivery of genetically modified hPDMSCs with the FGF2 and PDGF-BB genes into ischemic muscle has the ability to enhance neovascularization in a hindlimb ischemia model; and (iii) xenografted hPDMSCs survive in ischemic tissue for a minimum of 4 weeks subsequent to the initiation of cell therapy. The present study provides a novel therapeutical strategy for the treatment of ischemic diseases.

\section{Acknowledgments}

The current study was supported by the National Key Basic Research Program of China (2010 CB 529900) and the National High Technology Research and Development Program of China (2012AA020807).

\section{References}

1. Vincent KA, Shyu KG, Luo Y, et al: Angiogenesis is induced in a rabbit model of hindlimb ischemia by naked DNA encoding a HIF-1alpha/VP16 hybrid transcription factor. Circulation 102: 2255-2261, 2000.

2. Emanueli C, Salis MB, Pinna A, Graiani G, Manni L and Madeddu P: Nerve growth factor promotes angiogenesis and arteriogenesis in ischemic hindlimbs. Circulation 106: 2257-2262, 2002.
3. Luttun A, Tjwa M, Moons L, et al: Revascularization of ischemic tissues by PIGF treatment, and inhibition of tumor angiogenesis, arthritis and atherosclerosis by anti-Flt1. Nat Med 8: 831-840, 2002.

4. Dor Y, Djonov V, Abramovitch R, et al: Conditional switching of VEGF provides new insights into adult neovascularization and pro-angiogenic therapy. EMBO J 21: 1939-1947, 2002.

5. Asahara T, Bauters C, Zheng LP, et al: Synergistic effect of vascular endothelial growth factor and basic fibroblast growth factor on angiogenesis in vivo. Circulation 92 (Suppl): II365-II371, 1995.

6. Ieda Y, Fujita J, Ieda M, et al: G-CSF and HGF: Combination of vasculogenesis and angiogenesis synergistically improves recovery in murine hind limb ischemia. J Mol Cell Cardiol 42: 540-548, 2007.

7. Jay SM, Shepherd BR, Andrejecsk JW, Kyriakides TR, Pober JS and Saltzman WM: Dual delivery of VEGF and MCP-1 to support endothelial cell transplantation for therapeutic vascularization. Biomaterials 31: 3054-3062, 2010.

8. Lindner V, Lappi DA, Baird A, Majack RA and Reidy MA: Role of basic fibroblast growth factor in vascular lesion formation. Circ Res 68: 106-113, 1991.

9. van Royen N, Piek JJ, Buschmann I, Hoefer I, Voskuil M and Schaper W: Stimulation of arteriogensis; a new concept for the treatment of arterial occlusive disease. Cardiovasc Res 49: 543-553, 2001.

10. Raines EW: PDGF and cardiovascular disease. Cytokine Growth Factor Rev 15: 237-254, 2004.

11. Greenberg JI, Shields DJ, Barillas SG, et al: A role for VEGF as a negative regulator of pericyte function and vessel maturation. Nature 456: 809-813, 2008.

12. Cao R, Bråkenhielm E, Pawliuk R, et al: Angiogenic synergism, vascular stability and improvement of hind-limb ischemia by a combination of PDGF-BB and FGF-2. Nat Med 9: 604-613, 2003.

13. Hao X, Månsson-Broberg A, Gustafsson T, et al: Angiogenic effects of dual gene transfer of bFGF and PDGF-BB after myocardial infarction. Biochem Biophys Res Commun 315: 1058-1063, 2004

14. Cho SW, Moon SH, Lee SH, et al: Improvement of postnatal neovascularization by human embryonic stem cell-derived endothelial-like cell transplantation in a mouse model of hindlimb ischemia. Circulation 116: 2409-2419, 2007.

15. Enis DR, Shepherd BR, Wang Y, et al: Induction, differentiation, and remodeling of blood vessels after transplantation of Bcl-2-transduced endothelial cells. Proc Natl Acad Sci USA 102: 425-430, 2005.

16. Kinnaird T, Stabile E, Burnett MS, et al: Local delivery of marrow-derived sromal cells augments collateral perfusion through paracrine mechanisms. Circulation 109: 1543-1549, 2004.

17. Nagaya N, Kangawa K, Itoh T, et al: Transplantation of mesenchymal stem cells improves cardiac function in a rat model of dilated cardiomyopathy. Circulation 112: 1128-1135, 2005.

18. Ozawa K, Sato K, Oh I, et al: Cell and gene therapy using mesenchymal stem cells (MSCs). J Autoimmun 30: 121-127, 2008.

19. Cao Y, Sun Z, Liao L, Meng Y, Han Q and Zhao RC: Human adipose tissue-derived stem cells differentiate into endothelial cells in vitro and improve postnatal neovascularization in vivo. Biochem Biophys Res Commun 332: 370-379, 2005.

20. Matikainen T and Laine J: Placenta - an alternative source of stem cells. Toxicol Appl Pharmacol 207 (Suppl): 544-549, 2005.

21. Parolini O, Alviano F, Bagnara GP, et al: Concise review: isolation and characterization of cells from human term placenta: outcome of the first international Workshop on Placenta Derived Stem Cells. Stem Cells 26: 300-311, 2008.

22. Fukuchi Y, Nakajima H, Sugiyama D, Hirose I, Kitamura T and Tsuji K: Human placenta-derived cells have mesenchymal stem/progenitor cell potential. Stem Cells 22: 649-658, 2004.

23. Miao Z, Jin J, Chen L, et al: Isolation of mesenchymal stem cells from human placenta: comparison with human bone marrow mesenchymal stem cells. Cell Biol Int 30: 681-687, 2006.

24. Prather WR, Toren A, Meiron M, Ofir R, Tschope C and Horwitz EM: The role of placental-derived adherent stromal cell (PLX-PAD) in the treatment of critical limb ischemia. Cytotherapy 11: 427-434, 2009.

25. Kranz A, Wagner DC, Kamprad M, et al: Transplantation of placenta-derived mesenchymal stromal cells upon experimental stroke in rats. Brain Res 1315: 128-136, 2010. 
26. Ventura C, Cantoni S, Bianchi F, et al: Hyaluronan mixed esters of butyric and retinoic acid drive cardiac and endothelial fate in term placenta human mesenchymal stem cells and enhance cardiac repair in infarcted rat hearts. J Biol Chem 282: 14243-14252, 2007.

27. Nishishita T, Ouchi K, Zhang X, et al: A potential pro-angiogenic cell therapy with human placenta-derived mesenchymal cells. Biochem Biophys Res Commun 325: 24-31, 2004.

28. Shyu KG, Chang H and Isner JM: Synergistic effect of angiopoietin-1 and vascular endothelial growth factor on neoangiogenesis in hypercholesterolemic rabbit model with acute hindlimb ischemia. Life Sci 73: 563-579, 2003.

29. Cao Y, Cao R and Hedlund EM: Regulation of tumor angiogenesis and metastasis by FGF and PDGF signaling pathways. J Mol Med (Berl) 86: 785-789, 2008.

30. Millette E, Rauch BH, Defawe O, Kenagy RD, Daum G and Clowes AW: Platelet-derived growth factor-BB-induced human smooth muscle cell proliferation depends on basic FGF release and FGFR-1 activation. Circ Res 96: 172-179, 2005.

31. Rafii S and Lyden D: Therapeutical stem and progenitor cell transplantation for organ vascularization and regeneration. Nat Med 9: 702-712, 2003.

32. Tang J, Xie Q, Pan G, Wang J and Wang M: Mesenchymal stem cells participate in angiogenesis and improve heart function in rat model of myocardial ischemia with reperfusion. Eur J Cardiothorac Surg 30: 353-361, 2006.

33. Nishishita T, Ouchi K, Zhang X, et al: A potential pro-angiogenic cell therapy with human placenta-derived mesenchymal cells. Biochem Biophys Res Commun 325: 24-31, 2004.
34. Iwaguro H, Yamaguchi J, Kalka C, et al: Endothelial progenitor cell vascular growth factor gene transfer for vascular regeneration. Circulation 105: 732-738, 2002.

35. Phillips MI and Tang YL: Genetic modification of stem cells for transplantation. Adv Drug Deliv Rev 60: 160-172, 2008

36. Barbash IM, Chouraqui P, Baron J, et al: Systemic delivery of bone marrow-derived mesenchymal stem cells to the infracted myocardium: feasibility, cell migration, and body distribution. Circulation 108: 863-868, 2003.

37. Vulliet PR, Greeley M, Halloran SM, MacDonald KA and Kittleson MD: Intra-coronary arterial injection of mesenchymal stromal cells and microinfarction in dogs. Lancet 363: 783-784, 2004.

38. Liechty KW, MacKenzie TC, Shaaban AF, et al: Human mesenchymal stem cells engraft and demonstrate site-specific differentiation after in utero transplantation in sheep. Nat Med 6: 1282-1286, 2000.

39. Tsuji H, Miyoshi S, Ikegami Y, et al: Xenografted human amniotic membrane-derived mesenchymal stem cells are immunologically tolerated and transdifferentiated into cardiomyocytes. Circ Res 106: 1613-1623, 2010.

40. Ishii M, Numaguchi Y, Okumura K, et al. Mesenchymal stem cell-based gene therapy with prostacyclin synthase enhanced neovascularization in hindlimb ischemia. Atherosclerosis 206: $109-118,2009$ 